\title{
Lipid Polymorphisms and Membrane Shape
}

\author{
Vadim A. Frolov ${ }^{1,2,3}$, Anna V. Shnyrova ${ }^{1,2}$, and Joshua Zimmerberg ${ }^{4}$ \\ ${ }^{1}$ Unidad de Biofisica (Centro Mixto CSIC-UPV/EHU), Leioa 48940, Spain \\ ${ }^{2}$ Departamento de Biochimica y Biología Molecular, Universidad del Pais Vasco, Leioa 48940, Spain \\ ${ }^{3}$ IKERBASQUE, Basque Foundation for Science, 48011 Bilbao, Spain \\ ${ }^{4}$ Program in Physical Biology, Eunice Kennedy Shriver National Institute of Child Health and Human \\ Development, National Institutes of Health, Bethesda, Maryland 20892 \\ Correspondence: zimmerbj@mail.nih.gov
}

Morphological plasticity of biological membrane is critical for cellular life, as cells need to quickly rearrange their membranes. Yet, these rearrangements are constrained in two ways. First, membrane transformations may not lead to undesirable mixing of, or leakage from, the participating cellular compartments. Second, membrane systems should be metastable at large length scales, ensuring the correct function of the particular organelle and its turnover during cellular division. Lipids, through their ability to exist with many shapes (polymorphism), provide an adequate construction material for cellular membranes. They can selfassemble into shells that are very flexible, albeit hardly stretchable, which allows for their far-reaching morphological and topological behaviors. In this article, we will discuss the importance of lipid polymorphisms in the shaping of membranes and its role in controlling cellular membrane morphology.

Lseff ipids are natural molecules whose ability to self-assemble in dynamic macrostructures in water has been recognized as one of the important mechanisms of evolution (Luisi et al. 1999; Hanczyc and Szostak 2004). This ability is driven by the amphiphilic nature of lipid molecules which tend to aggregate so that the hydrophobic (tails) and the hydrophilic (heads) parts of the lipid are well separated, and the area of the dividing surface is held by the hydrophobic effect (Tanford 1980).

Because lipid assemblies "hate edges," they tend to form various closed structures or phases extended over the space, in which the average volumetric shape of lipid molecules is different in different phases. This plasticity in molecular shape was discovered in the first X-ray diffraction structural determinations of lipid/water mixtures under different osmotic stresses, and the investigators used the appropriate term from crystallography, polymorphism, to describe the "crystallization into two or more chemically identical but crystallographically distinct forms" (Random House Dictionary, 2nd ed.) (Luzatti et al. 1968). Amongst many of such structures, the bilayer plays the most important role in our life. Besides forming the core of cellular membranes, it is quickly gaining

Editor: Kai Simons

Additional Perspectives on The Biology of Lipids available at www.cshperspectives.org

Copyright (C) 2011 Cold Spring Harbor Laboratory Press; all rights reserved; doi: 10.1101/cshperspect.a004747

Cite this article as Cold Spring Harb Perspect Biol 2011;3:a004747 
V.A. Frolov et al.

technological popularity. Lipid capsules or closed bilayer vesicles are widely used in cosmetics, drug delivery, etc., primarily because of their natural biological compatibility (Hafez and Cullis 2001). These vesicles can be constructed from lipids or similar amphiphiles that sense the environment, temperature (through thermal expansion), $\mathrm{pH}$ (through protonation of the heads), and so on, so that they can be destabilized and release their content at the target place (Hafez and Cullis 2001). Lipid sacs can also have different shapes (such as spherical, polyhedral, and tubular vesicles) which may be important for the flow properties of vesicular suspensions (Florence et al. 2004). Hence, the main technological interest resides in making the lipid vesicle shape and topology switchable (i.e., the regulation of the shape of the lipid bilayer).

Living cells have to solve the same task on a daily basis. Cellular membranes are extremely dynamic, as cells (and biological matter in general) are in constant flux. This motion has both constitutive aspects that tend toward homeostasis, and regulated aspects that are governed by signaling networks. Consequently, cellular membranes preserve their morphology at large scale, forming organelles with distinct shape and function, whereas at smaller scales the membranes are constantly remodeled to maintain dynamic material exchange between different membrane formations (Paiement and Bergeron 2001; McMahon and Gallop 2005). This remodeling, however, may not interfere with the barrier function and the overall structural stability of the membranes. This combination of flexibility and the resistance against rupture is brought by the lipid bilayer. Despite the extreme protein crowding, the lipid bilayer remains the structural core of cellular membranes. The protein area occupancy in membranes generally does not exceed 20\% (Dupuy and Engelman 2008), so that the mean distance between membrane-inserting parts of the proteins is $\sim 10 \mathrm{~nm}$, more than 10 lipid molecules (Phillips et al. 2009). Thus, lipids "glue" the membrane components together and in the same time actively participate in membrane remodeling (Chernomordik and Kozlov 2003).
To balance dynamics and stability, cells employ different lipid species in the membrane (Dowhan 1997). Although the core phospholipids, such as PC, assemble stable lipid bilayers at physiological temperatures, many of cellular lipids intrinsically destabilize bilayers and, when purified, do not form bilayer phases at physiological conditions (Cullis and de Kruijff 1979). Such "nonbilayer" lipids as PE and DAG, however, are important membrane constituents: they mediate proteolipid interactions within the lipid bilayer (Dowhan 1997; Lee 2004; Ces and Mulet 2006) and increase morphological plasticity of the lipid bilayer (Hafez and Cullis 2001). The behavior of individual lipid species can be regulated: charged lipid species, such as PS, can switch its "bilayer" identity at elevated $\mathrm{pH}$ or $\mathrm{Ca}^{2+}$ (Hafez and Cullis 2001; Fuller et al. 2003; Zimmerberg et al. 2006). Nevertheless, the overall lipid mixture of cells is always balanced to form metastable lipid bilayers. Microorganisms living at different temperatures alter the ratio between bilayer and nonbilayer lipids dependently on the growth temperature (de Kruijff 1997). Besides dynamic regulation of the bulk lipid composition, cells specifically create nonuniform distribution of lipids within membrane organelles. First, in most of the membrane systems, except endoplasmic reticulum (van Meer et al. 2008), there is substantial compositional asymmetry between the two monolayers: for example, in plasma membrane such active lipid species as PS and PE are accumulated in the monolayer facing the cytoplasm (Hafez and Cullis 2001; Janmey and Kinnunen 2006; van Meer et al. 2008). Second, lipids and proteins can segregate laterally by forming domains of distinct compositions and functions (Lingwood et al. 2009). These three factors, large amounts of nonbilayer lipids, trans-bilayer asymmetry, and lateral interactions and segregation of protein and lipid species in the cellular membranes, provide a basis for the active involvement of lipids in cellular morphogenesis, as we describe below.

The morphological activity of the lipid bilayer is regulated by special enzymes maintaining or changing the lipid distribution in the membrane. This way, the lipid content of 
the membrane can be matched to the cellular "morphological" needs and to the physiological function of a particular membrane compartment, making lipids the mediators of the structure/function relationships at the organelle level (Shnyrova et al. 2009). Some fundamental principles linking the molecular identity of lipid species and their function are embedded into the molecular architecture of the lipids. Therefore, lipids can be designed (synthesized, metabolized, or intracellularly remade) to have a predictable morphological behavior.

\section{MOLECULAR ORGANIZATION OF LIPID POLYMORPHISM}

Forces Driving Self-Assembly of Lipid Bilayers

Although the intrinsic wish of lipids to avoid direct exposure of the oily tails to water can be intuitively understood, this wish can be fulfilled in various ways. The result will be determined by a complex balance of repulsive and attractive forces between lipid molecules in the lipid monolayer. Figures 1 and 2 illustrate this notion by using the concept of bilayer pressure $(\pi)$

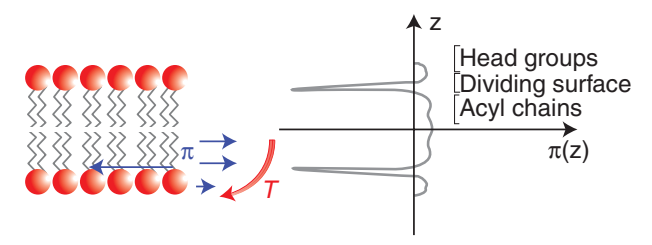

Figure 1. Lateral pressure profile across the lipid bilayer. For each monolayer, three clear regions can be distinguished. In the head-group region, a repulsive component from the entropic, steric, and electrostatic interactions is dominant. The hydrophobic effect, which holds the membrane together, manifests itself as a strong attractive force (negative pressure values) in the head/tail dividing surface. Finally, there is a repulsive component from the entropic and steric interactions in the acyl chains region. At equilibrium, the lateral force balance sets the pressure integral zero. However, uneven distribution of the positive and negative pressure over $z$ (illustrated by blue arrows) leads to the integral torque intending to bend a monolayer (red arrow). In symmetric case, the torques in two monolayers cancel each other resulting in the flat bilayer configuration. profile, which determines the redistribution of stresses across the lipid bilayer (Marsh 2007).

By looking at the cross section of the lipid bilayer, several distinct regions dominated by the proper set of forces become noticeable. The most important region for the structural stability of the monolayer is the one near the interface dividing the hydrophilic and hydrophobic parts of the lipids. Even a small increase in the area of this dividing surface leads to hydrophobic tail exposure, a process that is very costly energetically. Thus, the lipid monolayers are hardly expandable as lipids are held together by the hydrophobic effect resulting from the high negative pressure peaked at the dividing surface (Fig. 1). Changes in the dividing surface affect the membrane morphology the most so that this region is a general target for proteins that control membranes via hydrophobic insertion (Campelo et al. 2008).

For a "relaxed" lipid monolayer at equilibrium, with no external forces applied, the lateral force balance requires the pressure integral $\int_{-h / 2}^{h / 2} \pi d z$ to be zero. Thus the condensing effect of negative pressure in the dividing surface is balanced by the repulsive interactions in both the head-group and tail regions. Let us consider the forces existing in the head-group membrane region. Although, attractive hydrogen bonding and electrostatic attractions are present, the steric and electrostatic repulsions dominate in these areas of the lipid bilayer. The lipid heads are the ones most susceptible for external perturbations brought by the environment bathing the membrane. Hence, changes in the polar heads often result in imbalances in the pressure profile that interfere with the stability and morphology of the lipid bilayer. The most receptive are charged head groups whose electrostatic response depends on the $\mathrm{pH}$ and ionic composition of the bathing solution. Specialized protein domains, such as $\mathrm{PH}$ or ENTH domains, have been evolved to specifically recognize inositol head groups, such domains are commonly found in proteins controlling membrane remodeling in cells (Lemmon 2003).

As regards to the acyl chain region, it is the core of the lipid bilayer that consists of the tightly packed yet highly mobile lipid tails 
V.A. Frolov et al.

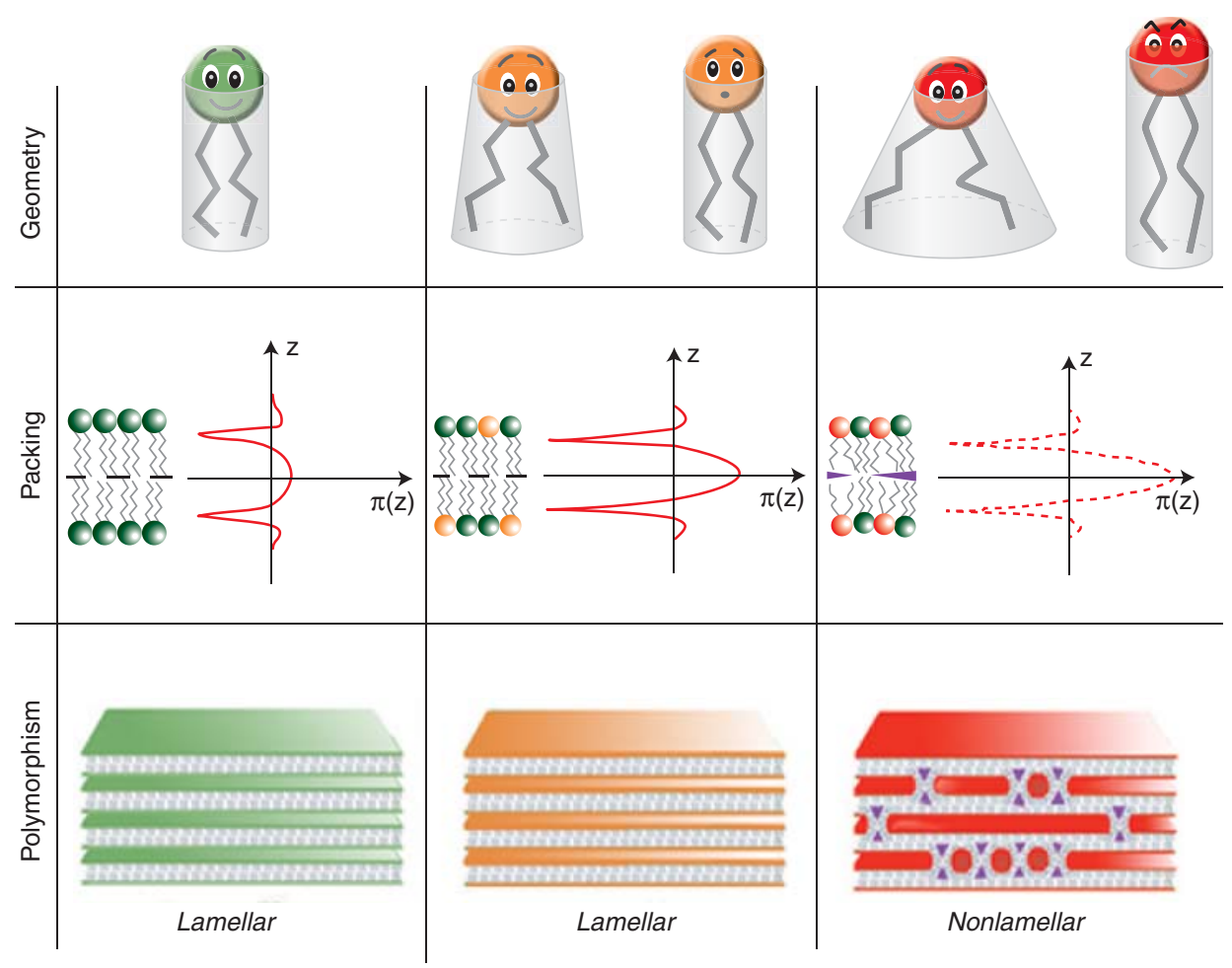

Figure 2. Molecular geometry of lipids and membrane stored stress. Monolayers made of cylindrical molecules of zero SC can form nonstressed lamellas (first column, green lipid). However, for nonzero SC, lipid molecules have to be reshaped to fit into a flat state, leading to membrane stress (second column, orange lipid). When the packing parameter of the lipid is too far from unity, the stress accumulated in the resulting bilayer is too big; hence the transition of the lamella into a nonlamellar (e.g., $\mathrm{H}_{\mathrm{II}}$ ) phase is favorable. The transition begins when small interlammellar contacts having characteristic hourglass shape form (third column, red lipid), lipids with negative SC promotes formation of these localized nonbilayer structures.

resembling a fluid phase. Here again there is an overall repulsive force which is a balance between attractive weak van der Waals forces and repulsive entropic forces, each of which depend on the tail chemistry and their interactions (Marsh 2007). Cellular membranes display considerable variety of acyl chain combinations, controlled by specialized enzymes (Dowhan 1997; van Meer et al. 2008). The specific enzymes acting on the lipid tails, such as phospholipases (PLAs), are intimately involved in creation of membrane morphology (Brown et al. 2003).

As mentioned above, at equilibrium the lateral force balance requires the pressure integral to be zero. However, the torque $\left(T \sim \int_{-h / 2}^{h / 2} \pi z d z\right)$ might remain unbalanced in each monolayer, and if so, it tends to bend the lipid monolayer (Fig. 1). The torque reflects the pressure distribution along the membrane normal. For bulkier tails the large entropic repulsion force is distributed over the whole tail region, so that the bearing point is shifted upward to the heads' part; the resulting torque tends to bend the monolayer (Fig. 1). For a symmetrical bilayer made of two equivalent monolayers the torques act in the opposite directions intending to tear the bilayer apart. However, creating a hydrophobic void inside a bilayer cost substantial energy (Gruner 1989), thus up to a critical value the torque results in creation of elastic stress stored in the flat bilayer. The amount of the stored stress depends on the lipids' "molecular shape," characterized by 
Lipid Polymorphisms and Membrane Shape

the relative bulkiness of their head groups and tails. Let us analyze this in detail.

\section{Lipid Molecular Shape and Membrane Stored Stress}

To parameterize the concepts of lipid molecular shape, one assigns a specific packing parameter $P(P=V / a l$, where $V$ is the specific volume occupied by the tails, $a$ is the area per lipid molecule in the dividing surface, and $l$ is the effective length of the tails' region) to lipids of certain "shape" (Israelachvili 1992). As in the fluid state, where lipids rotate freely, $P$ describes a shape with revolution symmetry; that is, $P \sim$ 1 to cylinders (e.g., dioleoylphosphocholine or DOPC), $P>1$ to cones (e.g., dioleoylphosphotidylethanolamine or DOPE), and $P<1$ to inverse cones (e.g., lysophosphocholine or LPC). Importantly, $P$ characterizes a situation of zero monolayer torque, when the curvature of the monolayer surface corresponds to the intrinsic wishes of the lipids. This curvature, termed spontaneous curvature (SC) or intrinsic curvature, characterizes the intrinsic shape of the monolayer of zero bending stress (Gruner 1985). Naturally occurring cellular lipids have a very wide range of spontaneous curvatures (from diacylglycerol (DAG) [Leikin et al. 1996] to LPC [Fuller and Rand 2001]) owing to the great variety of the fatty acids available for the acyl chain combinations. The SC of a multicomponent monolayer is well approximated by the sum of the SCs of all of its components (Kumar 1991). This additivity indicates that lipid tails may be considered as a uniform fluid-like oily phase whose density is similar to that of a 3D fluid mixture of the corresponding fatty acids (e.g., May et al. 2004).

Monolayers made of cylindrical molecules of zero SC can form nonstressed bilayers (Fig. 2, first column). However, for nonzero SC, lipid molecules have to be reshaped to fit into a flat state (Fig. 2, second and third column). A fundamental constraint here is that the tails' volume $V$ should remain constant during such reshaping because of fluid-like nature of the tails' region. We note here that the simple "oil phase" approximation works the best for the fluid-disordered lamella phase $\left(\mathrm{L}_{\mathrm{d}}\right)$, where, as in bulk fluid phase, there is no long-range correlation between mobility of the lipid tails (Mouritsen and Jorgensen 1994). In this article, we mostly focus on the $L_{d}$ phase as the basis for understanding the morphological dynamics of cellular membranes. If $V$ is to remain constant, the transformation of the conically shaped lipid into a cylindrical one will cause the averaged stretching or compressing of the acyl tails (Fig. 2 , orange and red geometries), which leads to membrane stress. The amount of stress is indicated by how far the $P$ is from unity, many cellular lipids, such us DAG and DOPE, destabilize bilayer (lamellar) state at physiological conditions, as they tend to form nonbilayer structures.

\section{Nonbilayer Lipid Structures}

The transformation of the lipid bilayer (lamellar phase) into nonbilayer structures (phases) is the classical manifestation of lipid polymorphism (de Kruijff 1997). The transformation is driven by the stored stress, which is augmented with temperature as it increases the repulsive pressure in the tail region and, thus, the bending torque. At a certain point the stored stress exceeds a critical value and a new phase nucleates (Fig. 2, third column) which frees the stressed lipid by placing it in an environment with less torque. For positive $P$, various nonbilayer "inverse" phases form, characterized by periodic arrangement of curved lipid monolayers, illustrating the rich landscape of lipid polymorphism (de Kruijff 1997; Shearman et al. 2006).

For the inverse hexagonal $\left(\mathrm{H}_{\mathrm{II}}\right)$ phase, monolayers are arranged in long cylinders whose geometry directly reports the SC when the coupling between cylinders is relaxed by the addition of a hydrocarbon solvent (Rand et al. 1990). The SC of many biologically important lipids, such as DAG or DOPE, fits an $\mathrm{H}_{\mathrm{II}}$ arrangement much better than a flat bilayer; because of the excess of stored stress they alone do not form any lamellar phase. Studies of the pathway of lamellar to $\mathrm{H}_{\mathrm{II}}$ transition provided the first conjecture on why cells need nonbilayer lipids at all: this transition is nucleated through localized connections between two 
V.A. Frolov et al.

closely apposed bilayers, mimicking membrane fusion events (Fig. 2, third column). These connections are characterized by a high monolayer curvature suitable for nonbilayer lipids. As the formation of such connections from cylindrical lipids is much more energetically costly, the intimate involvement of nonbilayer lipids in topological membrane remodeling, fusion, and fission was predicted and later confirmed experimentally (reviewed in Chernomordik et al. 1987; Chernomordik and Kozlov 2003). Consequently, though nonbilayer phases are not present widely in vivo, nonbilayer lipids play a key role as morphological elements required to support the dynamic organization of cellular membrane systems. We propose the term "lipid morphogen" to capture this idea that the stresses described above are embodied into the stressed lipid that then organizes membrane structure during topological transformations in biology.

\section{Membrane Asymmetry and Curvature}

Besides producing the various lyotropic phases described above, lipid polymorphism manifests itself in the morphological complexity shown by individual lipid lamella (bilayers) made of monolayers with different properties. In this case, the stored stress is unevenly distributed between the monolayers and a new pathway for the membrane stored stress relaxation emerges. The simplest example illustrating the consequences of an asymmetric stress distribution is a bilayer made of monolayers of different SC (Fig. 3). In the flat configuration, the bending torque is nonbalanced even at small stresses, but equilibrium can be achieved via membrane bending. Even when one monolayer is not stressed, the stresses in the opposite one cause the bilayer to bend. This is because of monolayer coupling (Sheetz and Singer 1974), as we described above, the formation of voids inside the bilayer is energetically prohibited. Hence a trans-membrane asymmetry, or differences in the elastic properties of the two monolayers, is a requirement for the emergence of bilayer curvature.

In this case, the spontaneous curvature of an asymmetric lipid bilayer is the result of a force balance between the intrinsic wishes of the

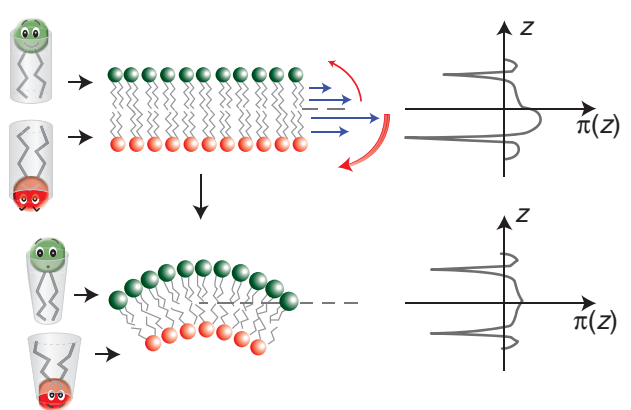

Figure 3. Curvature emergence through trans-membrane asymmetry. Lipid bilayers made of two different monolayers generally have an asymmetric pressure profile, the difference in the pressure distribution along the membrane normal $z$ (indicated by blue arrows) leads to the different monolayer torques (red arrows), the monolayer with the bigger torque "wins," and the bilayer bends to restore the torque balance.

two monolayers, each having the specific SC and bending resistance, dictated by the lipid composition. Although the range of SC in phospholipids is sufficiently large, their bending resistance is mainly independent on their SC (Fuller and Rand 2001). Hence, in most cases, the SC of the bilayer is defined by the sum of SC of its monolayers (similarly to the SC of each of the monolayers, dictated by the sum of the SC of their lipids).

The energy scale for the bending of a lipid bilayer from its spontaneous state is set mostly by the lipid tails. During any deformation, the spatial constraints imposed on the tails change and the corresponding repackaging energy can be estimated by simple Flory analysis (Rawicz et al. 2000). Far more sophisticated approaches are needed to account for great variety of experimentally observed phenomena (see Marrink et al. 2009), yet for small deformations of the lipid molecule the associated work can be estimated by a simple quadratic approximation (implemented by W. Helfrich): $E_{\mathrm{b}} \sim 2 k_{\mathrm{c}}$ $\left(C-C_{0}\right)^{2}$, where $C$ is the mean curvature of the dividing surface (equals to 0 for symmetric monolayer), $C_{0}$ is the SC, and $k_{\mathrm{c}}$ is the bending modulus which is a material constant characterizing the flexibility of particular tails. Measurements of $k_{\mathrm{c}}$ and the characterization of its lipid dependence have been approached in numerous 
Lipid Polymorphisms and Membrane Shape

studies using different methodologies (Marsh 2006). In agreement with the general "oil phase" approximation of the bilayer interior, $k_{\mathrm{c}}$ varies only moderately with lipid composition, with $25 k_{\mathrm{B}} T$ being the rough median (Marsh 2006). Shortening of lipid tails and introduction of double bonds soften the bilayer, whereas tails ordering and condensation increase $k_{\mathrm{c}}$ by several fold (Henriksen et al. 2006; Marsh 2006; Marsh 2007; Bashkirov et al. 2008). Nevertheless, the energies and the associated forces remain notably low: lipid bilayers are extremely bendable. The softness of the lipid bilayer is indicated by large temperature-driven fluctuations of the shape of freely suspended membrane objects (e.g., Dobereiner et al. 2003). This softness, combined with high structural stability, is crucial importance for the biological functionality of lipid bilayer. However, we acknowledge here that the bending rigidity increases dramatically with lateral ordering of the tails in fluid-ordered (Oradd et al. 2009) and crystalline bilayers, so that those phases are much less prone for morphological and topological remodeling.

To summarize, lipid bilayers possess extreme morphological plasticity because of small $k_{\mathrm{c}}$ and a wide variety of SC. Thus lipids can be considered as small elastic blocks which molecular shape is ultimately revealed at large scale via the self-assembly process. Although these blocks can be easily assembled into curved patches of lipid bilayer (Fig. 3B), such patches are yet to be pieced together to form a stable membrane configuration. Its shape and stability will depend on how the bilayer edges, which presence was neglected in the above analysis, are concealed from exposure to water.

\section{Closed Membranes and Shape Diagrams}

In most cases the stationary membrane formations are closed structures with no open edges. Edges, such as pores or localized breaches in membrane monolayers, form only transiently during topological remodeling of membranes or pathological processes. The shape of the closed membrane bilayer, such as a vesicle, is determined not only by the local force balance between membrane monolayers, but also by nonlocal factors.

One of these nonlocal factors is related to the difference in area between the inner and outer monolayers of the bilayer (Dobereiner et al. 1997). As monolayers are hardly expandable, changes in the area difference between the two lead to bending because of the bilayer coupling. In lipid vesicles, this effect is seen as membrane budding. The budding can be induced by temperature or addition of lipids to the outer monolayer (i.e., by factors changing the monolayers area balance), in this case because of relative expansion of the outer monolayer (Dobereiner et al. 1997; Papadopulos et al. 2007). To compensate, the vesicle is transformed into a chain of smaller vesicles to match the new area disparity. With time, lipid flip-flop can diminish the area difference and the membrane shape may relax to the initial spherical configuration.

Other nonlocal factors include lateral membrane tension and pressure differences between the vesicle interior and exterior. Because of the nonlocal nature of these factors, the shape of closed membrane systems is determined via global minimization of the elastic energy, where the effects of tension, pressure and nonlocal elasticity is accounted for via the corresponding Lagrange multipliers. Theoretical and experimental studies performed mostly on giant unilamellar vesicles (GUVs) have revealed complex shape diagrams (by analogy the with phase diagrams) of closed lipid bilayers (Lipowsky 1995; Dobereiner et al. 1997; Majhenc et al. 2004). The rich morphology of closed membrane shapes illustrates another dimension of lipid polymorphism displayed in closed lamellas. The extreme responsiveness of the morphology of lipid vesicles to various external perturbations provides the basis of the morphological activity of lipids in cellular systems.

\section{CHANGING MEMBRANE MORPHOLOGY}

As we have described above, lipids themselves can assemble into a wide variety of stable morphologies. Yet it is apparent that external energy sources are required to make those 
V.A. Frolov et al.

morphologies alive (i.e., to support dynamic transformations of the membrane shape). Proteins use different strategies to transform the energy (available from conformational changes or nucleotide hydrolysis) into membrane deformations. All these strategies had to evolve in tight cooperation with lipids, as the latter define the energy landscape for the protein action. Mechanistically, lipid-protein cooperation is implemented in two principal modes (Zimmerberg and Kozlov 2006). Proteins can perform "tuning" of membrane shape by matching the membrane composition and morphology. Here membrane shape is ultimately determined by lipids and membrane asymmetry. Alternatively, proteins like molecular motors or scaffolds can directly "force" membrane remodeling. Here, as the recent experiments have shown, the final shape is function of membrane elasticity (Bashkirov et al. 2008). Therefore, the effect of lipids is directly linked to the two main constituencies of lipid polymorphism: molecular shape and elasticity. Multiple experimental observations confirm the crucial involvement of lipid polymorphisms in membrane remodeling performed by various protein machines.

\section{Forcing Shape}

The high morphological plasticity of the fluid $\left(\mathrm{L}_{\mathrm{d}}\right)$ lipid bilayer is seemingly indicative of its low resistance to deformations imposed by proteins. However, at biologically relevant curvatures and length-scales this high compliance is not obvious: the curvatures of cellular tubulovesicular compartments reach high values and the amount of proteins participating in membrane remodeling is restricted by the size of the membrane objects and membrane crowding. Whenever direct force measurements become possible in reconstituted systems, the results show that protein action is in scale with membrane resistance, meaning that the proteins do not dominate the elastic resistance of lipid bilayer in determining overall membrane structure. This scaling can be illustrated in several examples.

One of the typical events in membrane remodeling is the formation of a thin tube from a flat lipid bilayer. The force which is required to pull a tube from a GUV (Koster et al. 2005) or plasma membrane (Sun et al. 2005) is on the order of tens of picoNewtons. This force is set by the membrane tension and bending rigidity $\left(F=2 \pi \sqrt{2 k_{\mathrm{c}} \sigma}\right)$ and thus can be further increased by augmenting the lateral membrane tension and/or the bending rigidity. Already for typical tensions and bending rigidity, whose ratio determines the characteristic curvature scale here $\left(r=\sqrt{k_{\mathrm{c}} / 2 \sigma}\right)$, the pulling force is comparable with those produced by few molecular motors (Shaklee et al. 2008). Changing $k_{\mathrm{c}}$ will give rise to the different tube geometry and will require different amount of motors to pull the tube. This way creation of a tubular geometry can be performed by motors combined with lipids.

The same similarity is noticeable when the specific (per unit area) energy of the tube formation is compared with the energy available from polymerization of proteins which squeeze the tube by forming a cylindrical scaffold on its surface, such as dynamin, or push the tube by forming a thin filament inside it, such as actin or tubulin (Shnyrova et al. 2009). The polymerization energy per protein unit ranges from $\sim 1 k_{\mathrm{B}} T$ for actin (Footer et al. 2007) to $4-10$ $k_{\mathrm{B}} T$ for tubulin and dynamin (Dogterom et al. 2005; Bashkirov et al. 2008; Roux et al. 2010), well corresponding to the specific elastic energy for the tubes of tens of $\mathrm{nm}$ in diameter. Again, increasing the elastic resistance of the tube membrane leads to a different final geometry (Bashkirov et al. 2008) illustrating the proteolipid cooperativity described above.

Finally, the energy required to make a spherical vesicle can be estimated as $500 k_{\mathrm{B}} T$ independently on the vesicle radius as it scales with area: $E=8 \pi k_{\mathrm{c}}$. In turn, if the vesicle is formed by a set of coat proteins and adaptors, the available energy will depend on the coat size. The two energies crossover when the vesicle diameter approaches $100 \mathrm{~nm}$ (Dobereiner et al. 1993; Nossal 2001) indicating, once again, protein-lipid cooperativity at a physiologically relevant length scale.

The above energy estimates assume "direct forcing" of membrane shape, where the stresses 
induced by an external force are uniformly distributed over the lipid molecules. However, proteins further cooperate with lipids to optimize the deformation process: They minimize the elastic stresses by tuning the membrane composition to the morphological demand (Fig. 4).

\section{Tuning of Membrane Shape \\ Creating Membrane Asymmetry}

As described above, membrane asymmetry is the driving force of the morphological transformations of a lipid bilayer. Lipid head groups are the part of the monolayer most susceptible for external perturbations. The repulsive interactions between the negatively charged lipids (such as phosphatidylserine (PS) or cardiolipin (CL) depend on the protonation of the charged residues. Neutralization of the charge in CL by applying acidic $\mathrm{pH}$ weakens the electrostatic repulsion and causes the decrease of the effective area CL occupies in a monolayer (Khalifat et al. 2008). Consequently, localized application of acidic $\mathrm{pH}$ to a GUV containing CL leads to inward membrane invagination and formation of tubes mimicking the mitochondrias cristae. In turn, expansion of the PS-containing monolayer by application of basic $\mathrm{pH}$ leads to outward invagination and rapid growth of the membrane protrusion (Fournier et al. 2009). Specialized proteins involved in membrane remodeling generally have strong affinity to charged lipids. Such proteins might cause similar changes in lipid head groups which might impact the membrane curvature creation (Zimmerberg et al. 2006).

Localized changes in membrane composition can also be produced by enzymes mediating lipid metabolism and interconvertion in cells. Some of them (e.g., PLA 2 ) are critically involved in membrane trafficking (Brown et al. 2003) and creation of cellular membrane morphologies (Christiansson et al. 1985). PLA (Staneva et al. 2004) and sphingomyelinase (Holopainen et al. 2000) morphological activity have been reconstituted with GUVs. These enzymes produce lipids with opposite packing parameter: Lysolipids are the by-product of
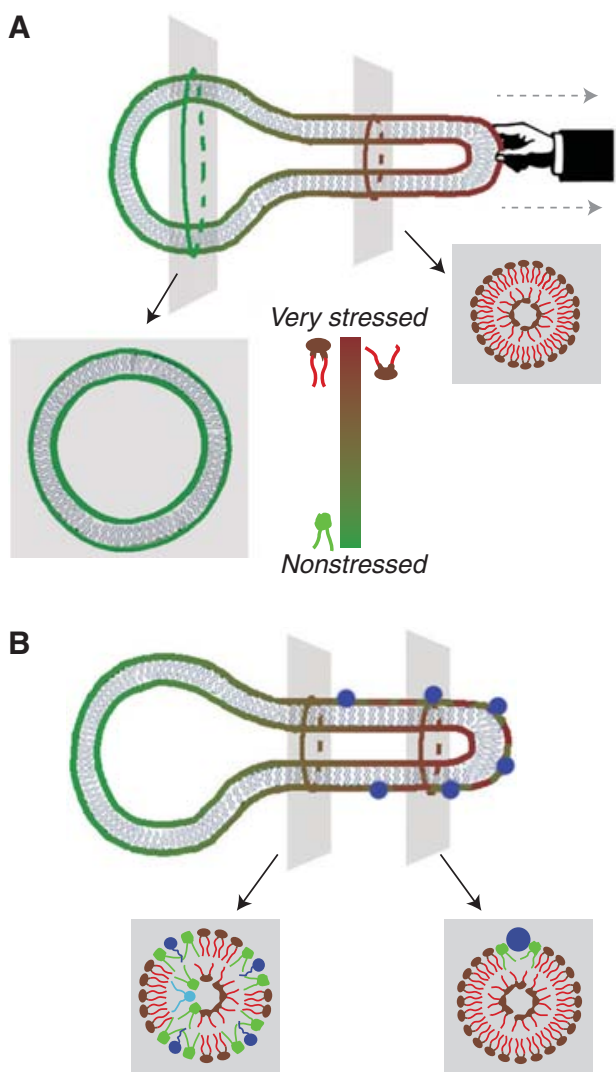

Figure 4. Forcing and tuning of the membrane shape. (A) Pulling a cylindrical membrane tube from a spherical vesicle by an external force; the force creates the high elastic stress (red) in the cylindrical regions, and the stress is also evident from dramatic transformation of lipid molecules in the cylinder seen in the red cross section. (B) The force-induced stress can be relaxed by tuning molecular composition of the membrane to its shape: Lipids with conical shape (left cross section) or shallow insertion of a protein domain (right cross section) relax the neighbor lipid molecules and diminish the integral elastic stress.

$\mathrm{PLA}_{2}$ activity, whereas sphingomyelinase convert sphingomyelins to ceramides. Accordingly, the opposite membrane curvatures have been produced by these enzymes in GUV. Furthermore, the activity of these enzymes is, in turn, modulated by nonbilayer lipids (Urbina et al. 2010).

Nevertheless, the curvature effect was not a direct consequence of the production of 
V.A. Frolov et al.

lipids with opposite SC. The enzyme adsorption causes changes in the area of the outer monolayer (Papadopulos et al. 2007). The reaction products, such as ceramides, tend to aggregate forming rigid membrane domains (Holopainen et al. 2000). Thus the morphological activity of the enzymes relies not only on the localized changes in membrane asymmetry, but also on nonlocal elastic response and domain-driven membrane morphology (see below).

In principle, membrane asymmetry can also be caused by clustering of lipids with certain spontaneous curvature. Asymmetric clustering of cholesterol by proteins orchestrating membrane vesiculation, such as viral matrix protein (Shnyrova et al. 2007) or caveolin (Martin and Parton 2005), can contribute to the creation of the vesicle morphology. More interestingly, such clustering can be promoted by the membrane curvature itself.

\section{Curvature-Composition Coupling}

On the basic level, the composition-morphology link corresponds to the curvature-coupling hypothesis (Leibler 1986). The coupling is revealed as lateral redistribution of lipids or lipid-protein complexes in a curved membrane so that the SC of the lipid bilayer approaches its geometric curvature (Sorre et al. 2009; Bashkirov et al. 2010; Capraro et al. 2010). This redistribution leads to lowering of the membrane energy and thus "auto-tuning" of membrane shape (Fig. 4B). Furthermore, the feedback between curvature and composition are multiple. Enzyme activity (Urbina et al. 2010), protein insertion and polymerization all are depend on curvature (McMahon and Gallop 2005; Drin and Antonny 2010; Roux et al. 2010). These effects are also coupled to component demixing in heterogeneous membranes (Sorre et al. 2009).

Hence, both the initiation of membrane transformation and the final membrane geometry depends on the coupling between spontaneous and geometric curvatures, opening a new "lateral" dimension for lipid polymorphism (Shnyrova et al. 2009). However, clear examples of lipid-driven morphology in vivo are yet to be revealed.

\section{Protein Insertion}

The current paradigm supported by numerous theoretical and experimental results states that hydrophobic insertion is the most powerful tool in creation of membrane curvature (Zimmerberg and Kozlov 2006; Campelo et al. 2008). Most of the protein machinery mediating membrane remodeling in cells rely on membrane-inserting domains, its critical involvement in formation of membrane tubes and vesicles as well as in topological membrane remodeling, fusion, and fission has been well established (reviewed in McMahon and Gallop 2005; Zimmerberg and Kozlov 2006). The basis of the insertion action is evident from the pressure profile: It forces a hydrophobic wedge inside the lipid bilayer acting against the attractive pressure (Fig. 4) and thus pushes lipids away. Yet the curvature activity of the insertion depends on how it modifies the pressure profile (Zemel et al. 2005; Campelo et al. 2008). The most effective are shallow insertions that penetrate slightly below the dividing surface and thus cause tilting of the tails of neighboring lipids (Fig. 4B). This tilt results in very high effective curvature of the proteolipid complex consisting of the inserting domain and its boundary lipids. For the ENTH domain of epsin, the adaptor protein is responsible for membrane curvature creation during endocytosis, this curvature being similar in magnitude to those created by DOPE or lysolipids (Capraro et al. 2010). The interaction between the protein inserting motifs and lipid polymorphism is evident from the experiments demonstrating the effect of membrane-inserting peptides on the phase behavior of lipids. And vice versa, lipids significantly modulate the insertion profile and the structure of the inserting protein domains (Marsh 2008). Thus, the curvature created by hydrophobic insertion is the result of proteolipid collaboration in which nonbilayer lipids and protein domains specialized on membrane remodeling are peers. Spatial coordination of multiple insertions by the scaffold proteins 
Lipid Polymorphisms and Membrane Shape

provides a general foundation of the shape creation in cells.

\section{Nonlocal Elasticity in Shape Tuning}

Besides changing the local membrane asymmetry, each insertion expands the lipid monolayer it inserts into. This expansion significantly impacts the shape transformation (Mui et al. 1995; Holopainen et al. 2000; Papadopulos et al. 2007). However, unlike changes in SC, this effect can be counterbalanced by lipid flipflop. Elegant experiments on GUV showed how the initial adsorption or insertion of a flippase into the outer monolayer of a GUV caused vesicle budding because of expansion of the outer monolayer. Subsequent increase in the flip-flop rate, caused by the actual flippase activity, led to shape restoration (Papadopulos et al. 2007).

Importantly, the same shape transformation can be performed by different means. An instructive example is the membrane tubulation or transition from a closed spherical shape to cylindrical shapes. Cylindrical geometry can be imposed by changes in SC of outer monolayer (proteins [e.g., synaptotagmin]; Martens et al. 2007) as well as by changes in the area difference between the inner and outer monolayers (Mui et al. 1995). Thus the hydrophobic insertion in combination with nonlocal elasticity and flip-flop provide a versatile set for the regulation of membrane morphology, essentially based on intrinsic polymorphic behavior of lipid bilayer vesicles.

\section{Domain-Driven Shape}

The final tool in lipid-driven membrane morphogenesis is provided via the ability of lipids to phase separate into fluid-coexistence regimes. The domain boundary provides the driving force for membrane budding even for a symmetric lipid bilayer. Domain-driven budding, predicted theoretically (Lipowsky 1992), has been studied in different lipid systems and the involvement of fluid domains in proteindriven membrane morphogenesis has been shown (Shnyrova et al. 2007, 2009). Does lipid polymorphism play any role in this process? SC of lipids becomes an important parameter in regulation of the main driving force of the domain-driven budding, the line tension (Dobereiner et al. 1993; Staneva et al. 2004). For most of the studied cases in which the fluid phase coexistence involves the so-called fluid ordered and fluid disordered phases, the thickness of the former is greater which leads to the membrane height mismatch at the phase boundary (Kuzmin et al. 2005). Nonbilayer lipids are ideally suited for lowering the energy of this mismatch by providing a smooth transition between membrane regions of different thickness. Thus, they accumulate in the boundary region and effectively lower the line tension. The stimulating effect of domain budding by lipids with large SC has been reconstructed experimentally (Dobereiner et al. 1993). In cells, it is now clear that a $100-\mathrm{nm}$ vesicle has a distinctly different lipid composition than its parent membrane, along with a different degree of lipid ordering (as determined by dyes), suggesting that phase separation and its consequent line tension between domains give rise both to budding and lipid sorting (Klemm et al. 2009). This efficient "boundary" effect plays a very important role in the latest class of membrane transformations involved in cellular membrane morphogenesis: topological remodeling.

\section{TOPOLOGICAL PLASTICITY OF MEMBRANES}

To preserve the compartmentalization of the intracellular space, lipid bilayers of cellular membranes must maintain their structural stability. Lipid lamellae made of lipids with small SC are stable formations under physiological conditions. They provide a foundation for the barrier function of cellular membranes and repel each other at close contact so that membrane vesicles do not merge spontaneously. However, cellular membranes contains sufficient amounts of nonbilayer lipids. Figure 2 shows how such lipids can promote localized destabilization of lamellar phase and formation of nonbilayer connections between the opposed lipid bilayers. This is the scenario for the 
V.A. Frolov et al.

involvement of lipid polymorphism in topological membrane remodeling driven by cellular proteins.

Cellular organelles are constantly exchanging material. This material transport and the morphology of the organelles rely of the balance of two fundamental processes, fusion and fission (Paiement and Bergeron 2001). Nonbilayer lipids make these processes energetically feasible as they lower the energy barrier of the nonbilayer intermediates structures to the level surmountable by the concerted action of specialized proteins (Chernomordik and Kozlov 2003). The structure of these nonbilayer intermediates, as well as the role of proteolipid cooperation and lipid polymorphism in membrane fusion and fission have been a focus of multiple reviews (Chernomordik et al. 1987; Chernomordik and Kozlov 2003; McMahon and Gallop 2005; Chernomordik et al. 2006). We emphasize here that the presence of nonbilayer lipids allows dynamic coupling between the membrane morphology, material transport and signaling networks, providing the basis of the dynamic organization of endomembrane system.

\section{CONCLUDING REMARKS}

We have seen how lipids are at the core of cellular morphogenesis. They are involved in regulation of morphological transformation at all levels; many of them are directly involved in intracellular signaling providing multiple levels of feedback between cellular morphology and metabolism. Perhaps the lipid bilayer was once an organizing platform on which the protein machineries, now governing the membrane behavior, had been built. More far out, we may speculate that lipids primogeniture explains many of the structural arrangements characteristic for those protein complexes conducting membrane fusion and fission, membrane-interacting domains, and protein scaffolds. The importance of the structural and geometrical plasticity of lipid bilayer in the various aspects of cellular life underlies the newly emerging multifaceted nature of lipid polymorphism.

\section{REFERENCES}

Bashkirov PV, Akimov SA, Evseev AI, Schmid SL, Zimmerberg J, Frolov VA. 2008. GTPase cycle of dynamin is coupled to membrane squeeze and release, leading to spontaneous fission. Cell 135: 1276-1286.

Bashkirov PV, Chekashkina K, Akimov SA, Frolov VA. 2010. Variation of membrane composition caused by strong bending. Biochemistry (Moscow) Suppl Ser A 5: 214-220.

Brown WJ, Chambers K, Doody A. 2003. Phospholipase A2 (PLA2) enzymes in membrane trafficking: mediators of membrane shape and function. Traffic 4: 214-221.

Campelo F, McMahon HT, Kozlov MM. 2008. The hydrophobic insertion mechanism of membrane curvature generation by proteins. Biophys J 95: 2325-2339.

Capraro BR, Yoon Y, Cho W, Baumgart T. 2010. Curvature sensing by the epsin N-terminal homology domain measured on cylindrical lipid membrane tethers. J Am Chem Soc 132: 1200-1201.

Ces O, Mulet X. 2006. Physical coupling between lipids and proteins: A paradigm for cellular control. Signal Transduction 6: 112-132.

Chernomordik LV, Kozlov MM. 2003. Protein-lipid interplay in fusion and fission of biological membranes. Annu Rev Biochem 72: 175-207.

Chernomordik LV, Melikyan GB, Chizmadzhev YA. 1987. Biomembrane fusion: A new concept derived from model studies using two interacting planar lipid bilayers. Biochim Biophys Acta 906: 309-352.

Chernomordik LV, Zimmerberg J, Kozlov MM. 2006. Membranes of the world unite! J Cell Biol 175: 201-207

Christiansson A, Kuypers FA, Roelofsen B, Op den Kamp JA, van Deenen LL. 1985. Lipid molecular shape affects erythrocyte morphology: A study involving replacement of native phosphatidylcholine with different species followed by treatment of cells with sphingomyelinase $\mathrm{C}$ or phospholipase A2. J Cell Biol 101: 1455-1462.

Cullis PR, de Kruijff B. 1979. Lipid polymorphism and the functional roles of lipids in biological membranes. Biochim Biophys Acta 559: 399-420.

de Kruijff B. 1997. Lipid polymorphism and biomembrane function. Curr Opin Chem Biol 1: 564-569.

Dobereiner HG, Evans E, Kraus M, Seifert U, Wortis M. 1997. Mapping vesicle shapes into the phase diagram: A comparison of experiment and theory. Phys Rev E 55: $4458-4474$.

Dobereiner HG, Gompper G, Haluska CK, Kroll DM, Petrov PG, Riske KA. 2003. Advanced flicker spectroscopy of fluid membranes. Phys Rev Lett 91: 048301.

Dobereiner HG, Kas J, Noppl D, Sprenger I, Sackmann E. 1993. Budding and fission of vesicles. Biophys $J$ 65: 1396-1403.

Dogterom M, Kerssemakers JW, Romet-Lemonne G, Janson ME. 2005. Force generation by dynamic microtubules. Curr Opin Cell Biol 17: 67-74.

Dowhan W. 1997. Molecular basis for membrane phospholipid diversity: why are there so many lipids? Annu Rev Biochem 66: 199-232.

Drin G, Antonny B. 2010. Amphipathic helices and membrane curvature. FEBS Lett 584: 1840-1847. 
Dupuy AD, Engelman DM. 2008. Protein area occupancy at the center of the red blood cell membrane. Proc Natl Acad Sci 105: 2848-2852.

Florence AT, Nasseri B, Arunothyanun P. 2004. Does shape matter? Spherical, polyhedral, and tubular vesicles. In Carrier-based drug delivery, pp. 75-84. American Chemical Society, Washington, DC.

Footer MJ, Kerssemakers JW, Theriot JA, Dogterom M. 2007. Direct measurement of force generation by actin filament polymerization using an optical trap. Proc Natl Acad Sci 104: 2181-2186.

Fournier JB, Khalifat N, Puff N, Angelova MI. 2009. Chemically triggered ejection of membrane tubules controlled by intermonolayer friction. Phys Rev Lett 102: 018102.

Fuller N, Rand RP. 2001. The influence of lysolipids on the spontaneous curvature and bending elasticity of phospholipid membranes. Biophys J 81: 243-254.

Fuller N, Benatti CR, Rand RP. 2003. Curvature and bending constants for phosphatidylserine-containing membranes. Biophys J 85: 1667-1674.

Gruner SM. 1985. Intrinsic curvature hypothesis for biomembrane lipid composition: a role for nonbilayer lipids. Proc Natl Acad Sci 82: 3665-3669.

Gruner SM. 1989. Stability of lyotropic phases with curved interfaces. J Phys Chem 93: 7562-7570.

Hafez IM, Cullis PR. 2001. Roles of lipid polymorphism in intracellular delivery. Adv Drug Deliv Rev 47: 139-748.

Hanczyc MM, Szostak JW. 2004. Replicating vesicles as models of primitive cell growth and division. Curr Opin Chem Biol 8: 660-664.

Henriksen J, Rowat AC, Brief E, Hsueh YW, Thewalt JL, Zuckermann MJ, Ipsen JH. 2006. Universal behavior of membranes with sterols. Biophys J 90: 1639-1649.

Holopainen JM, Angelova MI, Kinnunen PK. 2000. Vectorial budding of vesicles by asymmetrical enzymatic formation of ceramide in giant liposomes. Biophys $J$ 78: $830-838$.

Israelachvili J. 1992. Intermolecular and surface forces, pp. 366-389. Academic, Santa Barbara, CA.

Janmey PA, Kinnunen PK. 2006. Biophysical properties of lipids and dynamic membranes. Trends Cell Biol 16: 538-546.

Khalifat N, Puff N, Bonneau S, Fournier JB, Angelova MI. 2008. Membrane deformation under local $\mathrm{pH}$ gradient: Mimicking mitochondrial cristae dynamics. Biophys J 95: 4924-4933.

Klemm R, Ejsing CS, Surma M, Kaiser HJ, Gerl M, Sampaio JL, de Robillard Q, Ferguson C, Proszynski TJ, Shevchenko A, Simons K. 2009. Segregation of sphingolipids and sterols during formation of secretory vesicles at the trans-Golgi network. J Cell Biol 185: 601-612.

Koster G, Cacciuto A, Derenyi I, Frenkel D, Dogterom M. 2005. Force barriers for membrane tube formation. Phys Rev Lett 94: 068101.

Kumar VV. 1991. Complementary molecular shapes and additivity of the packing parameter of lipids. Proc Natl Acad Sci 88: 444-448.

Kuzmin PI, Akimov SA, Chizmadzhev YA, Zimmerberg J, Cohen FS. 2005. Line tension and interaction energies of membrane rafts calculated from lipid splay and tilt. Biophys J 88: 1120-1133.
Lee AG. 2004. How lipids affect the activities of integral membrane proteins. Biochim Biophys Acta 1666: 62-87.

Leibler S. 1986. Curvature instability in membranes. J Phys (Paris) 47: 507-516.

Leikin S, Kozlov MM, Fuller NL, Rand RP. 1996. Measured effects of diacylglycerol on structural and elastic properties of phospholipid membranes. Biophys J 71: 26232632.

Lemmon MA. 2003. Phosphoinositide recognition domains. Traffic 4: 201-213.

Lingwood D, Kaiser HJ, Levental I, Simons K. 2009. Lipid rafts as functional heterogeneity in cell membranes. Biochem Soc Trans 37: 955-960.

Lipowsky R. 1992. Budding of membranes induced by intramembrane domains. J Phys II France 2: 1825-1840.

Lipowsky R. 1995. The morphology of lipid membranes. Curr Opin Struct Biol 5: 531-540.

Luisi PL, Walde P, Oberholzer T. 1999. Lipid vesicles as possible intermediates in the origin of life. Curr Opinion Coll Interface Sci 4: 33-39.

Luzzati V, Tardieu A, Gulik-Krzywicki T. 1968. Polymorphism of lipids. Nature 217: 1028-1030.

Majhenc J, Bozic B, Svetina S, Zeks B. 2004. Phospholipid membrane bending as assessed by the shape sequence of giant oblate phospholipid vesicles. Biochim Biophys Acta 1664: 257-266.

Marrink SJ, de Vries AH, Tieleman DP. 2009. Lipids on the move: simulations of membrane pores, domains, stalks and curves. Biochim Biophys Acta 1788: 149-168.

Marsh D. 2006. Elastic curvature constants of lipid monolayers and bilayers. Chem Phys Lipids 144: 146-159.

Marsh D. 2007. Lateral pressure profile, spontaneous curvature frustration, and the incorporation and conformation of proteins in membranes. Biophys J 93: 3884-3899.

Marsh D. 2008. Protein modulation of lipids, and vice-versa, in membranes. Biochim Biophys Acta 1778: 1545-1575.

Martens S, Kozlov MM, McMahon HT. 2007. How synaptotagmin promotes membrane fusion. Science 316: $1205-$ 1208.

Martin S, Parton RG. 2005. Caveolin, cholesterol, and lipid bodies. Semin Cell Dev Biol 16: 163-174.

May S, Kozlovsky Y, Ben-Shaul A, Kozlov MM. 2004. Tilt modulus of a lipid monolayer. Eur Phys J E Soft Matter 14: $299-308$.

McMahon HT, Gallop JL. 2005. Membrane curvature and mechanisms of dynamic cell membrane remodelling. Nature 438: 590-596.

Mouritsen OG, Jorgensen K. 1994. Dynamical order and disorder in lipid bilayers. Chem Phys Lipids 73: 3-25.

Mui BL, Dobereiner HG, Madden TD, Cullis PR. 1995. Influence of transbilayer area asymmetry on the morphology of large unilamellar vesicles. Biophys $J$ 69: 930-941.

Nossal R. 2001. Energetics of clathrin basket assembly. Traffic 2: 138-147.

Oradd G, Shahedi V, Lindblom G. 2009. Effect of sterol structure on the bending rigidity of lipid membranes: A ${ }^{2} \mathrm{H}$ NMR transverse relaxation study. Biochim Biophys Acta 1788: $1762-1771$. 
V.A. Frolov et al.

Paiement J, Bergeron J. 2001. The shape of things to come: Regulation of shape changes in endoplasmic reticulum. Biochem Cell Biol 79: 587-592.

Papadopulos A, Vehring S, Lopez-Montero I, Kutschenko L, Stockl M, Devaux PF, Kozlov M, Pomorski T, Herrmann A. 2007. Flippase activity detected with unlabeled lipids by shape changes of giant unilamellar vesicles. J Biol Chem 282: 15559-15568.

Phillips R, Ursell T, Wiggins P, Sens P. 2009. Emerging roles for lipids in shaping membrane-protein function. Nature 459: 379-385.

Rand RP, Fuller NL, Gruner SM, Parsegian VA. 1990. Membrane curvature, lipid segregation, and structural transitions for phospholipids under dual-solvent stress. Biochemistry 29: 76-87.

Rawicz W, Olbrich KC, McIntosh T, Needham D, Evans E. 2000. Effect of chain length and unsaturation on elasticity of lipid bilayers. Biophys J 79: 328-339.

Roux A, Koster G, Lenz M, Sorre B, Manneville JB, Nassoy P, Bassereau P. 2010. Membrane curvature controls dynamin polymerization. Proc Natl Acad Sci 107: 4141-4146.

Shaklee PM, Idema T, Koster G, Storm C, Schmidt T, Dogterom M. 2008. Bidirectional membrane tube dynamics driven by nonprocessive motors. Proc Natl Acad Sci 105: 7993-7997.

Shearman GC, Ces O, Templer RH, Seddon JM. 2006. Inverse lyotropic phases of lipids and membrane curvature. J Phys: Condens Matter 18: S1105-S1124.

Sheetz MP, Singer SJ. 1974. Biological membranes as bilayer couples. A molecular mechanism of drug-erythrocyte interactions. Proc Natl Acad Sci 71: 4457-4461.

Shnyrova AV, Frolov VA, Zimmerberg J. 2009. Domaindriven morphogenesis of cellular membranes. Curr Biol 19: R772-R780.
Shnyrova AV, Ayllon J, Mikhalyov II, Villar E, Zimmerberg J, Frolov VA. 2007. Vesicle formation by self-assembly of membrane-bound matrix proteins into a fluidlike budding domain. J Cell Biol 179: 627-633.

Sorre B, Callan-Jones A, Manneville JB, Nassoy P, Joanny JF, Prost J, Goud B, Bassereau P. 2009. Curvature-driven lipid sorting needs proximity to a demixing point and is aided by proteins. Proc Natl Acad Sci 106: 5622-5656.

Staneva G, Angelova MI, Koumanov K. 2004. Phospholipase A2 promotes raft budding and fission from giant liposomes. Chem Phys Lipids 129: 53-62.

Sun M, Graham JS, Hegedus B, Marga F, Zhang Y, Forgacs G, Grandbois M. 2005. Multiple membrane tethers probed by atomic force microscopy. Biophys J 89: 4320-4329.

Tanford C. 1980. The hydrophobic effect: Formation of micelles and biological membranes, 2nd ed. Wiley, New York.

Urbina P, Flores-Diaz M, Alape-Giron A, Alonso A, Goni FM. 2010. Effects of bilayer composition and physical properties on the phospholipase $\mathrm{C}$ and sphingomyelinase activities of Clostridium perfringens $\alpha$-toxin. Biochim Biophys Acta 1808: 279-286.

van Meer G, Voelker DR, Feigenson GW. 2008. Membrane lipids: Where they are and how they behave. Nat Rev Mol Cell Biol 9: 112-124.

Zemel A, Ben-Shaul A, May S. 2005. Perturbation of a lipid membrane by amphipathic peptides and its role in pore formation. Eur Biophys J 34: 230-242.

Zimmerberg J, Kozlov MM. 2006. How proteins produce cellular membrane curvature. Nat Rev Mol Cell Biol 7: 9-19.

Zimmerberg J, Akimov SA, Frolov V. 2006. Synaptotagmin: Fusogenic role for calcium sensor? Nat Struct Mol Biol 13: 301-303. 


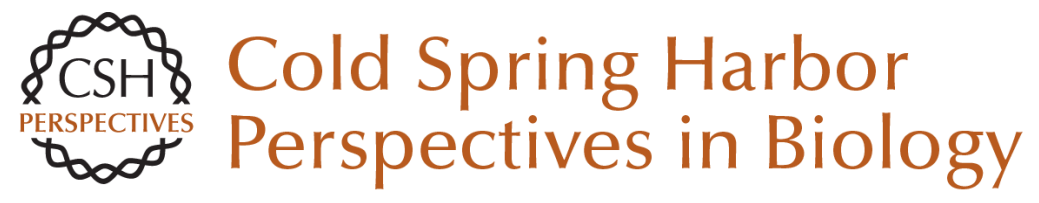

\section{Lipid Polymorphisms and Membrane Shape}

Vadim A. Frolov, Anna V. Shnyrova and Joshua Zimmerberg

Cold Spring Harb Perspect Biol 2011; doi: 10.1101/cshperspect.a004747 originally published online June 6, 2011

Subject Collection The Biology of Lipids

Role of Lipids in Virus Replication Maier Lorizate and Hans-Georg Kräusslich

Model Answers to Lipid Membrane Questions Ole G. Mouritsen

\section{Glycosphingolipid Functions} Clifford A. Lingwood

Regulation of Cholesterol and Fatty Acid Synthesis

Jin Ye and Russell A. DeBose-Boyd

Lipid-Mediated Endocytosis

Helge Ewers and Ari Helenius

Fluorescence Techniques to Study Lipid

Dynamics

Erdinc Sezgin and Petra Schwille

Lysosomal Lipid Storage Diseases

Heike Schulze and Konrad Sandhoff

Distribution and Functions of Sterols and Sphingolipids

J. Thomas Hannich, Kyohei Umebayashi and Howard Riezman
Membrane Organization and Lipid Rafts Kai Simons and Julio L. Sampaio

Shotgun Lipidomics on High Resolution Mass

Spectrometers

Dominik Schwudke, Kai Schuhmann, Ronny

Herzog, et al.

Glycosphingolipid Functions Clifford A. Lingwood

Phosphoinositides in Cell Architecture Annette Shewan, Dennis J. Eastburn and Keith Mostov

Synthesis and Biosynthetic Trafficking of Membrane Lipids Tomas Blom, Pentti Somerharju and Elina Ikonen

Lipid Polymorphisms and Membrane Shape Vadim A. Frolov, Anna V. Shnyrova and Joshua Zimmerberg

Specificity of Intramembrane Protein-Lipid Interactions

Francesc-Xabier Contreras, Andreas Max Ernst, Felix Wieland, et al.

Dynamic Transbilayer Lipid Asymmetry Gerrit van Meer

For additional articles in this collection, see http://cshperspectives.cshlp.org/cgi/collection/

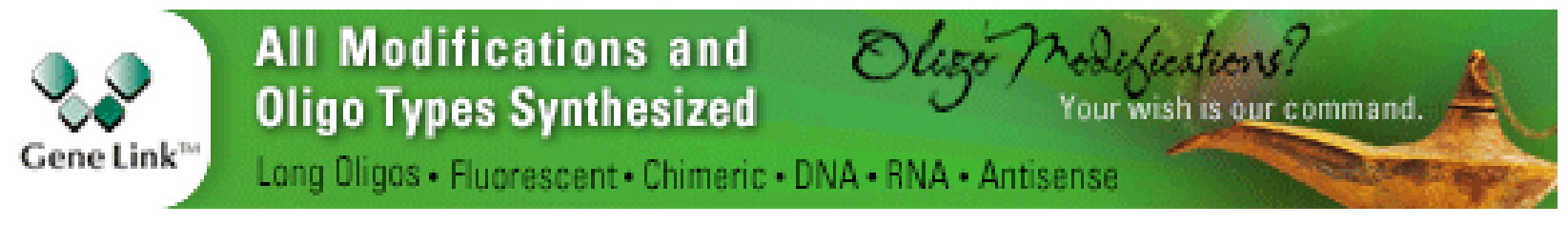

Copyright @ 2011 Cold Spring Harbor Laboratory Press; all rights reserved 